\title{
Evaluación de la funcionalidad del eje hipotálamo-hipófiso-adrenal a través del test de respuesta del cortisol al despertar en pacientes con diabetes mellitus tipo 1 con y sin depresión: Estudio Multicéntrico Argentino (EMA-1)
}

\author{
Evaluation of the hypothalamo-pituitary-adrenal axis using the cortisol \\ awakening response test in patients with type 1 diabetes mellitus with \\ and without depression: Multicenter Argentine Study (EMA-1)
}

\author{
Adriana Álvarez ${ }^{1,2}$, Andrea Kozak ${ }^{3}$, Lucas Costa ${ }^{4}$, Guillermo Alzueta ${ }^{1,5}$, Juan Bauchi ${ }^{1,6}$, Claudia De Boni ${ }^{1,7}$, \\ Guillermo Dieuzeide ${ }^{1,8}$, Olga Escobar ${ }^{1,9}$, Teresita García ${ }^{1,10}$, Patricia Mascaró ${ }^{1,11}$, Olguita Vera ${ }^{1,12}$, \\ Diego González ${ }^{13}$, Bibiana Fabre ${ }^{13}$, Luis Grosembacher ${ }^{14}$
}

\begin{abstract}
RESUMEN
Introducción: la depresión (DP) tiene una alta prevalencia en pacientes con diabetes mellitus tipo 1 (DM1) y se asocia a repercusiones clínicas negativas como mayor morbimortalidad cardiovascular y complicaciones crónicas. Existen pocos estudios publicados sobre la funcionalidad del eje hipotálamo-hipófiso-adrenal $(\mathrm{H}-\mathrm{H}-\mathrm{A})$ en DM1 con DP, y la relación entre la DP y el test de respuesta del cortisol al despertar (RCD) con el control glucémico (CG).

Objetivos: analizar la funcionalidad del eje $\mathrm{H}-\mathrm{H}-\mathrm{A}$ a través de la evaluación del RCD en pacientes con DM1 (PD1) con y sin DP. Como objetivos secundarios, conocer la prevalencia de DP en PD1 y ver si existe relación entre el RCD y CG, y entre DP y CG. Materiales y métodos: estudio observacional, prospectivo, de corte transversal, multicéntrico, nacional. Se incluyeron PD1 mayores de 18 años; se utilizó cuestionario Patient Health Questionnaire-9 (PHO-9) para diagnóstico de DP. Se tomaron muestras de cortisol salival al despertar y a los 30 minutos (RCD), y se consideró RCD bloqueado si el valor de cortisol de los 30 minutos no aumentaba más del $50 \%$ del basal. Además se tomaron muestras de sangre en ayunas para medir glucemia, fructosamina y $\mathrm{HbA} 1 \mathrm{c}$. Resultados: se incluyeron 79 pacientes, 39\% hombres, edad promedio $38 \pm 15$ años, duración de la diabetes de $16 \pm 13$ años; $53 \%$ casados/en pareja y $87 \%$ con ingresos económicos estables. El 68\% de los PD1 presentó el RCD bloqueado. En PD1 con DP el $85 \%$ presentó el RCD bloqueado vs el $60 \%$ en los no deprimidos y dicha diferencia fue marginalmente significativa $(p=0,05)$. La prevalencia de DP fue de 39\%. No se encontró ninguna relación significativa entre RCD bloqueado y control glucémico ( $p>0,05)$. Los PD1 con DP moderada-severa presentaron un peor control glucémico en relación a los PD1 sin depresión (evaluado por glucemia mayor de $120 \mathrm{mg} / \mathrm{dl}$, fructosamina mayor de $285 \mathrm{umol} / \mathrm{l} ; \mathrm{p}<0,05)$ y la relación no fue significativa para HbA1c aunque mostró una tendencia. Conclusiones: en pacientes con DM1 y DP se halló el RCD bloqueado en un alto porcentaje. Dado que la DP se asocia a mayor morbimortalidad cardiovascular, podría utilizarse el test de RCD como biomarcador de DP, y podría servir para estratificar esta sub-población de alto de riesgo. La depresión moderada-severa se asoció a peor control glucémico, por lo tanto, diagnosticar y tratar adecuadamente la DP en PD1 podría contribuir a prevenir la aparición o progresión de complicaciones crónicas.
\end{abstract}

Palabras clave: diabetes mellitus tipo 1; eje hipotálamo-hipófiso-adrenal; depresión; control glucémico.

\section{ABSTRACT}

Introduction: depression (DP) has a high prevalence in patients with type 1 diabetes mellitus (DM1) and is associated with negative clinicals consequences like more cardiovascular morbimortality and chronic complications. There are few studies published about the dysregulation of hypothalamopituitary-adrenal axis (H-P-A) in DM1 with DP and the relation between DP and the Cortisol Awakening Response Test (CAR) with the glycemic control (GC).

Objectives: examine the functionality of the H-P-A axis using the Cortisol Awakening Response Test (CAR), in patients with DM1 (PD1) with and without DP. Determine the prevalence of $D P$ in $P D 1$ and examine if there is any relation between CAR and GC and DP and poorer GC.

Materials and methods: observational, prospective, national, multicenter study. Patients with DM1, older than 18 years old; Patient Health-9 questionnaire (PHQ-9) was used to diagnose DP and 2 samples of salivary cortisol, and blood samples for glycemia, glycated albumin and Hba1C.

Results: 79 patients with DM1 (PD1) were included, 39\% male, mean age $38 \pm 15$ years old, an average of $16 \pm 13$ years evolution of diabetes; $53 \%$ married/couple and $87 \%$ have a regular incomes. $68 \%$ of PD1 presented CAR blunted. In PD1 with DP $85 \%$ has CAR blunted versus $60 \%$ in those without DP, and this difference was marginally significant $(p=0.05)$. The prevalence of $D P$ was $39 \%$. No significant relation was found between CAR blunted and glycemic control ( $p>0.05)$.PD1 with Moderate-severe DP showed worse metabolic control than the PD1 without DP (evaluated by glycemia higher than $120 \mathrm{mg} / \mathrm{dl}$, glycated albumin higher than 285 umol/l); $p<0.05$ ) and the relation was not significant with $\mathrm{HbA1C}$ but it showed a trend.

Conclusions: patients with DM1 and DP presented a high prevalence of CAR blunted. DP is related with higher cardiovascular morbi-mortality, thus CAR would be useful as a biomarker of DP and would be used to stratify this population of high risk. DP moderate-severe was related to worse glycemic control, hence diagnose and treat correctly DP in PD1 would contribute to prevent the onset or the evolution of chronic complications.

Key words: type1 diabetes mellitus; hypothalamus-pituitary-adrenal-axis; depression; glycemic control. 
1 Comité de Aspectos Psicosociales de la Sociedad Argentina de Diabetes, Ciudad Autónoma de Buenos Aires, Argentina

2 Médica de Planta del Servicio de Endocrinología, Metabolismo y Medicina Nuclear, Hospital Italiano de Buenos Aires, Ciudad Autónoma de Buenos Aires, Argentina

3 Bioquímica, Directora Técnica del Laboratorio de Endocrinología Hormonal y Genético del Servicio de Endocrinología, Metabolismo y Medicina Nuclear, Hospital Italiano de Buenos Aires, Ciudad Autónoma de Buenos Aires, Argentina

4 Bioingeniero, Unidad de Bioestadística, Facultad de Ciencias Médicas, Universidad Nacional del Litoral, Santa Fe, Argentina

5 Consultorio privado, Balcarce, Provincia de Buenos Aires, Argentina

6 Jefe del Servicio de Endocrinología, Hospital Naval Puerto Belgrano, Bahía Blanca, Provincia de Buenos Aires, Argentina

7 Consultorio privado, Mar del Plata, Provincia de Buenos Aires, Argentina

8 Jefe División Medicina Interna, Jefe del Servicio de Endocrinología y Diabetes, Hospital Ntra. Sra. Del Carmen, Chacabuco, Provincia de Buenos Aires, Argentina

9 Diagnos Centro Privado, Tucumán, Argentina

10 Consultorio Privado, Facultad de Medicina, Universidad Nacional de Tucumán, Tucumán, Argentina
1 Consultorio Privado, Comodoro Rivadavia, Chubut, Argentina

12 Consultorio Privado, Río Gallegos, Santa Cruz, Argentina

13 Bioquímico/a, Laboratorio de Endocrinología, Departamento de Bioquímica Clínica, Facultad de Farmacia y Bioquímica, Universidad de Buenos Aires, Ciudad Autónoma de Buenos Aires, Argentina

14 Médico de Planta, Servicio de Endocrinología, Metabolismo y Medicina Nuclear, Jefe de la Sección Diabetes, Hospital Italiano de Buenos Aires, Ciudad Autónoma de Buenos Aires, Argentina

Contacto de la autora: Adriana Álvarez

E-mail: adriana.alvarez@hospitalitaliano.org.ar Correspondencia: Camila O Gorman 373, Depto 1701

(C1107DEA) Puerto Madero, Ciudad Autónoma de Buenos

Aires, Argentina

Fecha de trabajo recibido: 07/07/20

Fecha de trabajo aceptado: 25/10/20

Conflictos de interés: los autores declaran que no existe conflicto de interés.

\section{INTRODUCCIÓN}

La diabetes mellitus (DM) es una enfermedad crónica y progresiva, asociada no sólo a complicaciones físicas, sino también psicológicas. En la actualidad se calcula que hay 463 millones de pacientes con DM y se proyecta que para el año 2045 habrá 700 millones de pacientes a nivel mundial según el atlas de la International Diabetes Federation 20191. Existen dos tipos de DM, siendo la más prevalente la de tipo 2 , que se caracteriza por afectar a personas adultas con obesidad y que cursa con mayor morbimortalidad de causa cardiovascular. La DM1 afecta sólo al 10\% de los pacientes, es predominantemente de causa autoinmune y el 50\% incluye a jóvenes menores de 30 años. Debido a la disminución de la masa de células beta, el único tratamiento posible es la administración de insulina, existiendo el riesgo de cetoacidocisis y coma ante la falta o el aumento de su requerimiento ${ }^{2}$.

La depresión (DP) asociada a la DM es una de las comorbilidades psicológicas más estudiadas dada su alta prevalencia ${ }^{3}$, y se caracteriza por estado de ánimo deprimido, anhedonia, disminución de la energía, sentimientos de culpa, alteraciones del sueño, del apetito y falta de concentración.

Dado que la DM y la DP afectan a más de 350 millones de personas cada una de ellas, se considera a ambas enfermedades como un problema de Salud Pública ${ }^{4-5}$.

En diferentes trabajos publicados se ha comunicado una mayor prevalencia de depresión mayor tanto en pacientes con DM2 como con DM1 en comparación con la población general ${ }^{6}$. Particularmente las mujeres con DM tienen dos a tres veces más riesgo de padecer $\mathrm{DP}^{7}$. Recientemente hemos publicado la prevalencia de DP en pacientes con DM2 y su alto nivel de sub-diagnóstico ${ }^{8}$. Los jóvenes con DM1 tienen tres veces más riesgo de presentar DP cuando se los compara con la población control ${ }^{9-10}$.

Se ha reportado la prevalencia de DP en pacientes con DM1 por Barnard et al. ${ }^{11}$ en una revisión sistemática de la literatura en pacientes adultos con DM1, y se halló un 12\% de depresión clínica en pacientes vs un 3,2\% en controles.

La asociación DP y DM tiene implicancias clínicas desfavorables como, por ejemplo, mayor morbimortalidad cardiovascular, disminución de la funcionalidad y de la calidad de vida, mayores costos de atención y disminución de la adherencia al tratamiento ${ }^{12-13}$.

Los mecanismos que subyacen a las altas tasas de DP en DM y sus efectos adversos aún no están íntegramente comprendidos, pero es probable que incluyan factores biológicos y psicológicos. Un modelo teórico de origen psicológico, considerando que hay muchos, no es suficiente para explicar la alta prevalencia y las consecuencias biológicas de la DP en DM. Varios estudios encontraron correlación entre DP y mal control glucémico en pacientes con $\mathrm{DM}^{14}$.

Alteraciones hormonales y un estado inflamatorio subclínico son dos factores biológicos que 
están presentes en ambas enfermedades. La presencia de dichos factores podría explicar algunas de las implicancias clínicas de la comorbilidad. Este tema ha sido muy investigado en DM2, pero poco se sabe sobre los mecanismos etiopatogénicos de la DP en DM1 ${ }^{15}$. Recientemente Herder et al. publicaron una asociación entre citokinas inflamatorias (IL-1RA y PCR) y síntomas depresivos en un estudio realizado en 389 pacientes con DM1 ${ }^{16}$.

La activación crónica del eje hipotálamo-hipófiso-adrenal $(\mathrm{H}-\mathrm{H}-\mathrm{A})$ con aumento del cortisol puede producir efectos adversos como hiperglucemia, osteoporosis y aumento de la enfermedad cardiovascular. Pacientes con DM y complicaciones crónicas presentan niveles elevados de cortisol y éstos se relacionan con la severidad de dichas complicaciones ${ }^{17}$. También hay evidencia sobre la disfuncionalidad del eje $\mathrm{H}-\mathrm{H}-\mathrm{A}$ en pacientes con $\mathrm{DM}^{18}$. Existe abundante evidencia sobre las alteraciones del eje $\mathrm{H}-\mathrm{H}-\mathrm{A}$ en pacientes con DP, pero generalmente se presentan en casos más severos como la depresión de tipo melancólica. Recientemente se ha publicado que en pacientes con DM1 y DP, los niveles de cortisol nocturno se hallan alterados y son influidos por distintos factores como el tipo de DP, el tabaquismo y la estación del año ${ }^{19}$.

Una manera de evaluar la funcionalidad del eje $\mathrm{H}-\mathrm{H}-\mathrm{A}$ es a través del test de respuesta del cortisol al despertar (RCD), conocido por su sigla en inglés CAR (cortisol awakening response), que consiste en tomar una muestra basal de cortisol salival 5 minutos ( $\mathrm{min}$ ) luego de despertar y otra a los 30 min, considerándose normal a un aumento del cortisol salival a los 30 min de un $50 \%$ por encima del basal20.

Otra manera de evaluar el eje $\mathrm{H}-\mathrm{H}-\mathrm{A}$ es a través de la curva diurna de cortisol, que se calcula restando al cortisol salival nocturno el cortisol salival matinal ${ }^{21}$.

La hiperglucemia per se puede alterar el eje $\mathrm{H}-\mathrm{H}-\mathrm{A}$, como se ha publicado en el estudio KORA-F321y en el Whithehall $\|^{22}$, donde ambos trabajos hallaron niveles más altos de cortisol salival nocturno en pacientes con DM2 comparados con grupo control.

\section{OBJETIVOS}

Dado que a la fecha existen pocos trabajos publicados en relación a las alteraciones del eje hipotálamo-hipófiso-adrenal en la población adulta con DM1 (PD1) y DP'19, el objetivo primario del presente trabajo es evaluar la funcionalidad del eje
$\mathrm{H}-\mathrm{H}-\mathrm{A}$ a través del test de RCD en pacientes con DM1 con y sin DP. Como objetivos secundarios, conocer la prevalencia de DP en PD1, y detectar si existe relación entre $R C D$ y con el control glucémico (CG), y entre DP y CG.

\section{MATERIALESY MÉTODOS Diseño, ámbito del estudio y población}

Se realizó un estudio observacional, prospectivo, de corte transversal, multicéntrico, nacional, aprobado por el Comité de Ética del Hospital Italiano de Buenos Aires, entre marzo de 2016 y diciembre de 2018. El ámbito de investigación correspondió a centros de atención de diabetes de las ciudades de Buenos Aires, Balcarce, Bahía Blanca, Mar del Plata, Tucumán, Comodoro Rivadavia y Río Gallegos. Basándonos en los recursos disponibles para ejecutar el estudio, se empleó un tamaño muestral a conveniencia de 79 pacientes con DM1. Los pacientes incluidos fueron aquellos que consultaron consecutivamente en los centros de investigación intervinientes durante el período descripto previamente, y que luego de firmar consentimiento informado, ingresaban al estudio.

Los criterios de inclusión fueron: 1) diagnóstico clínico previo de DM1 realizado por un especialista en diabetes; 2) edad: mayor de 18 años; 3) haber concurrido para atención diabetológica en los últimos 12 meses a los centros intervinientes; 4) firma del Consentimiento Informado (protocolo aprobado por el Comité de Ética de Protocolos de Investigación, CEPI).

Los criterios de exclusión fueron: 1) enfermedad mental grave; 2) consumo de corticoides; 3) hipotiroidismo descompensado; 4) patología grave no asociada a la diabetes; 5) consumo de antidepresivos hasta 15 días previos.

Se descartó la presencia de enfermedades mentales severas por los antecedentes de la historia clínica y la ausencia de medicación previa con psicofármacos.

\section{Definiciones y recolección de variables}

Dado que en todos los centros diabetológicos no se contaba con la presencia de Médicos especialistas en Salud Mental, para realizar el diagnóstico de depresión se utilizó la versión en español de la escala de depresión PHQ-9 (Patient Health Questionnaire) según fue diseñada por Kroenke et al. ${ }^{23}$. La misma es de autollenado, consta de nueve ítems y es una escala de tipo Likert con cuatro puntos que evalúa la presencia de síntomas de- 
presivos (correspondientes a los criterios DSM-IV) presentes en las últimas dos semanas e incluye los siguientes criterios:

1) Síndrome depresivo mayor: si hay por lo menos 5 ítems puntuados al menos como "más de la mitad de los días" o "casi todos los días", y al menos uno de los ítems positivos corresponde al 1 ó 2 (ánimo depresivo o anhedonia).

2) Otro síndrome depresivo: presencia de dos, tres o cuatro síntomas depresivos por "más de la mitad de los días", y que uno de los síntomas sea ánimo depresivo o anhedonia.

Un reciente estudio poblacional basado en una cohorte que incluyó pacientes con DM2 recomendó utilizar como punto de corte para diagnóstico de DP a través del PHQ-9 un puntaje de 5, ya que mostró la mejor sensibilidad (92,3\%) con especificidad aceptable $(70,4 \%)^{24}$.

La severidad se estableció según la siguiente puntuación: leve de 5-9, y moderado-severo si el puntaje era mayor a 9, un criterio que se utiliza para estudios de investigación ${ }^{25}$ dado que en la práctica clínica Petrak et al. recomiendan utilizar un puntaje mayor a $15^{26}$. La versión en español del PHQ-9 fue validado en Latinoamérica ${ }^{27}$.

Se definió la presencia de una complicación crónica cuando existía al menos una de las siguientes entidades: enfermedad cardiovascular $(E C)$, retinopatía diabética (RD), nefropatía diabética (ND) diagnosticada por presencia de microalbuminuria o proteinuria o elevación de la creatinina o disminución del clearence de creatinina medidos dichos parámetros en laboratorio local, y neuropatía periférica (presencia de síntomas como parestesias, hipoestesia, dolor y/o test alterado de monofilamento y/o electromiografía patológica). Todos estos datos se recolectaron de la historia clínica. Buen control glucémico se consideró a una $\mathrm{A} 1 \mathrm{c}$ igual o menor a 7,5\%, fructosamina igual o menos a 285 umol/l y una glucemia en ayunas igual o menor a $120 \mathrm{mg} / \mathrm{dl}$. El día del estudio, y luego de haber leído y firmado el Consentimiento Informado, se confeccionó una ficha clínica con datos recabados de la historia clínica. Se realizó examen físico que constó en medir peso y talla para calcular el índice de masa corporal, se tomó la tensión arterial a nivel braquial con tensiómetro de mercurio con la persona sentada luego de 5 min de reposo; se realizaron tres mediciones y se sacó el promedio. Los pacientes concurrieron en ayunas y se tomaron muestras de sangre para la medición de glucemia (medida por método enzimático UV hexoquinasa), HbA1c (medida por método HPLC o inmunoturbidimetría Tina-quant de Roche, valor de referencia: 5-5,8\%) y fructosamina (método NBT tetrazolio, valor de referencia: 205-285 umol/l).

Se dieron instrucciones para la toma de muestra en saliva en domicilio; al despertar (en ayunas y sin cepillarse los dientes) sólo podían beber agua y a los 30 min del basal la segunda muestra de saliva para la medición de cortisol. Se guardaron en frío y se entregaron al laboratorio al día siguiente. El cortisol salival se midió por quimioluminiscencia (Siemmens), límite de detección 1,0 nmol/L, sensibilidad analítica $1,0 \mathrm{nmol} / \mathrm{L}$, valor de referencia cortisol matinal: 4,7 a 32,0 nmol/L.

Se indagó sobre estado de convivencia, ingresos mensuales, obra social o pre-pago y años de escolaridad. Se recopilaron datos demográficos y clínicos.

\section{Análisis estadístico}

Los análisis estadísticos se realizaron con IBM SPSS v23.0. Las variables cuantitativas se describieron como media como medida de centralidad y desvío estándar como medida de dispersión. Las variables cualitativas se definieron con frecuencias absolutas y sus correspondientes porcentajes. Las comparaciones de proporciones se realizaron con la prueba de chi cuadrado de Pearson o con el test exacto de Fisher dependiendo de la distribución de valores esperados. El nivel de significancia seleccionado fue $\alpha=0,05$.

\section{Consideraciones éticas}

Este estudio fue aprobado por el Comité de Ética de Protocolos de Investigación del Hospital Italiano de Buenos Aires. Todos los participantes firmaron consentimiento antes de ingresar al estudio. Los costos de la investigación fueron cubiertos con un subsidio especial otorgado en 2015 por la Sociedad Argentina de Diabetes al Comité de Aspectos Psicosociales de la SAD.

\section{RESULTADOS}

Se incluyeron 79 pacientes con DM1, 39\% $(n=31)$ hombres, edad promedio $38 \pm 15$ años, con $16 \pm 13$ años de duración de su diabetes. Se obtuvieron variables demográficas, clínicas y antropométricas cuyos valores se detallan en la Tabla 1.

El test de RCD (cortisol salival matinal al despertar y cortisol salival a los 30 min post-desper- 
tar) pudo evaluarse en 60 pacientes. Se consideró RCD bloqueado cuando el valor del cortisol de 30 min post despertar no aumentaba por encima de un $50 \%$ del valor basal. El 68\% $(n=41)$ de los PD1 presentó el RCD bloqueado (Gráfico 1). En PD1 con DP el $85 \%(n=17)$ tenía el RCD bloqueado vs el $60 \%(n=24)$ en los no deprimidos, dicha diferencia fue marginalmente significativa $\left(\chi^{2}=3,85\right.$; g.l.=1; $p=0,05$ ) (Gráfico 2). El sexo, la edad, el nivel educativo, las complicaciones crónicas y la duración de la diabetes no fueron factores predictores de RCD bloqueado ( $>>0,05)$. Al comparar proporciones de RCD bloqueado y las variables del control glucémico tampoco se hallaron relaciones estadísticamente significativas, glucemia de ayuno $>120 \mathrm{mg} / \mathrm{dL}\left(\chi^{2}=0,72 ; \mathrm{g} . \mathrm{I} .=1 ; p=0.39\right)$, fructosamina $>285 \mathrm{mmol} / \mathrm{l}\left(\chi^{2}=0,92 ; \mathrm{g} . \mathrm{l} .=1 ; p=0,33\right)$ y A1c $>7,5 \%\left(\chi^{2}=0,16 ; g . l .=1 ; p=0,69\right)$.

El 39\% $(n=31)$ de los PD1 presentó DP (PHQ-9 $\geq 5)$, donde el $28 \%(n=22)$ tenía DP leve (PHQ-9: $5-9)$ y el $11 \%(n=9)$ DP moderada-severa (PHQ-9 >9) (Gráfico 3).

En la Tabla 2 se observa que dos de las variables asociadas al control glucémico (CG) sí mostraron una asociación estadísticamente significativa con la DP moderada-severa (DPM/S) y la tercera mostró una tendencia hacia la significancia, presentando el grupo con mal CG (según glucemia de ayuno mayor a $120 \mathrm{mg} / \mathrm{dL}$ ) un $28 \%$ de pacientes con DPM/S contra un $3 \%$ en los que tenían un buen CG $\left(\chi^{2}=9,15 ; g . I .=2 ; p=0,01\right)$. La fructosamina elevada (mayor a $285 \mathrm{mmol} / \mathrm{l}$ ) tuvo una frecuencia de pacientes con DPM/S de $15 \%$ vs $7 \%$ en los que tenían fructosamina normal, siendo dicha diferencia estadísticamente significativa $\left(\chi^{2}=8,51\right.$; g.I. $=2 ; p=0,014)$. Los PD1 con A1c menor a 7,5\% presentaron una frecuencia nula (0\%) de DPM/S vs un $17 \%$ de DPM/S en los que tenían A1c mayor a $7,5 \%$ y esa diferencia no fue significativa aunque mostró una tendencia $\left(\chi^{2}=5,2 ; g . l .=2 ; p=0,07\right)$.

\begin{tabular}{|c|c|}
\hline Perfil demográfico & \\
\hline Edad $(\bar{x} \pm D E)$ & $38 \pm 15$ \\
\hline Sexo masculino+ $\mathrm{N}(\%)$ & $39(49)$ \\
\hline Residencia urbana $\mathrm{N}(\%)$ & $75(95)$ \\
\hline Educación universitaria $\mathrm{N}(\%)$ & $47(59)$ \\
\hline Casados/pareja N (\%) & $42(53)$ \\
\hline Ingreso estable $\mathrm{N}(\%)$ & $69(87)$ \\
\hline Cobertura obra social N (\%) & $56(71)$ \\
\hline \multicolumn{2}{|c|}{ Perfil diabetológico, clínico y antropométrico } \\
\hline Años de diabetes $(\bar{x} \pm \mathrm{DE})$ & $16 \pm 13$ \\
\hline Glucemia $(\bar{x} \pm D E)$ & $128 \pm 61$ \\
\hline Fructosamina $(\bar{x} \pm \mathrm{DE})$ & $355 \pm 95$ \\
\hline $\mathrm{HbA1c}(\bar{x} \pm \mathrm{DE})$ & $8,3 \pm 1,5$ \\
\hline Retinopatía N (\%) & $16(20)$ \\
\hline Neuropatía N(\%) & $8(10)$ \\
\hline Nefropatía N(\%) & $16(20)$ \\
\hline Enfermedad cardíaca N (\%) & $4(5)$ \\
\hline Hipertensión arterial $\mathrm{N}(\%)$ & $20(25)$ \\
\hline Dislipemia N (\%) & $15(19)$ \\
\hline Fumador activo $\mathrm{N}(\%)$ & $13(17)$ \\
\hline Ejercicio diario $\mathrm{N}(\%)$ & $31(39)$ \\
\hline IMC $(\bar{x} \pm D E)$ & $24,3 \pm 3,5$ \\
\hline
\end{tabular}

$\bar{x}$ : media. DE: desvío estándar.

Tabla 1: Descripción del perfil de la muestra total disponible.

\begin{tabular}{|c|c|c|c|c|}
\hline & Sin depresión & DPL & $\mathrm{DPM} / \mathrm{S}$ & \\
\hline & N (\%) & N (\%) & $\mathrm{N}(\%)$ & Valor P* \\
\hline \multicolumn{5}{|c|}{ Glucemia } \\
\hline$\leq 120$ & $23(74)$ & $7(23)$ & $1(3)$ & \multirow{2}{*}{0,01} \\
\hline$>120$ & $10(40)$ & $8(32)$ & $7(28)$ & \\
\hline \multicolumn{5}{|c|}{ Fructosamina } \\
\hline$\leq 285$ & $13(93)$ & $0(0)$ & $1(7)$ & \multirow{2}{*}{0,014} \\
\hline$>285$ & $20(50)$ & $14(35)$ & $6(15)$ & \\
\hline \multicolumn{5}{|c|}{ HbA1c } \\
\hline$\leq 7,5$ & $18(75)$ & $6(25)$ & $0(0)$ & \multirow{2}{*}{0,07} \\
\hline$>7,5$ & $29(56)$ & $14(27)$ & $9(17)$ & \\
\hline
\end{tabular}

*Valores p correspondientes a pruebas Chi cuadrado de Pearson. En negritas los valores $p$ estadísticamente significativos. DPL: depresión leve; DP M/S: depresión moderada/severa.

Tabla 2: Comparación de perfil depresivo frente a variables de control glucémico. 


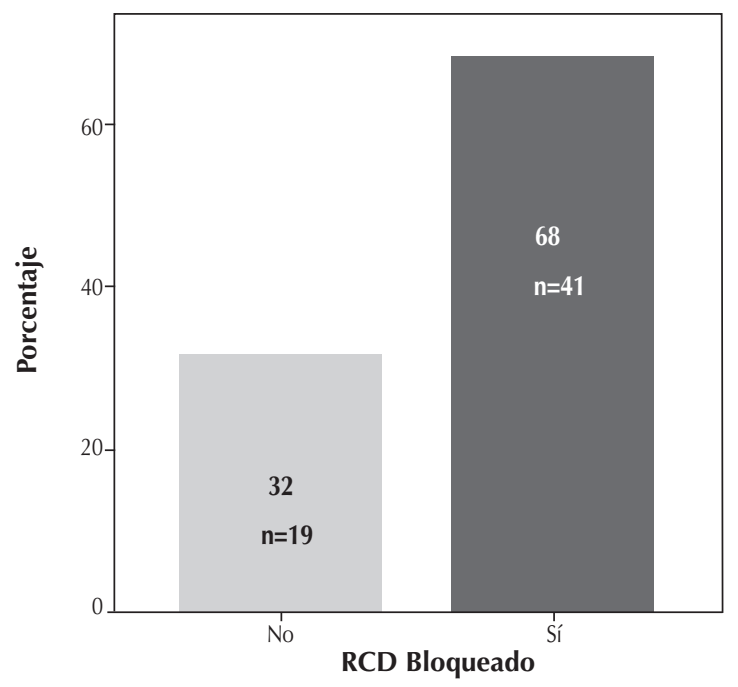

Gráfico 1: Distribución de frecuencias de RCD bloqueado.

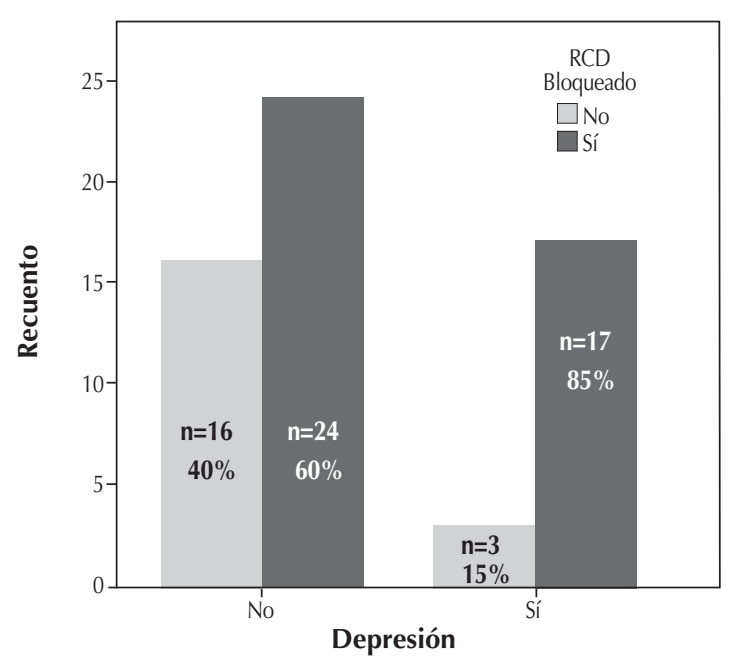

Gráfico 2: Comparación de proporción de RCD bloqueado entre grupos de depresión.

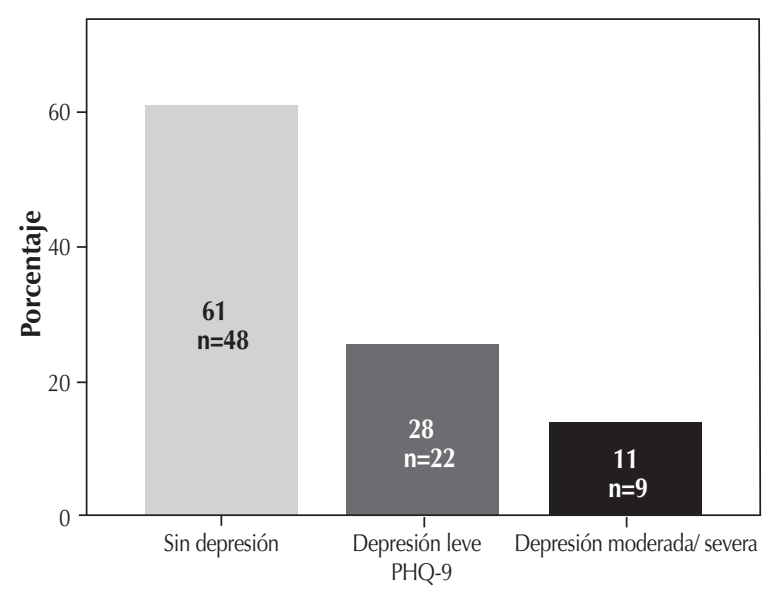

Gráfico 3: Distribución de frecuencias de categorías de depresión según cuestionario $\mathrm{PHO}-9$.

\section{DISCUSIÓN}

La depresión aumenta en 1,5 veces el riesgo de muerte por causa cardiovascular y por todas las causas tanto en pacientes con DM2 como con DM1. Esto fue demostrado por van Dooren et al. en un metaanálisis de estudios relevantes longitudinale ${ }^{28}$.

Múltiples conexiones biológicas podrían explicar dicha asociación, entre ellas el aumento de citoquinas proinflamatorias, alteraciones del eje $\mathrm{H}-\mathrm{H}-\mathrm{A}$, alteraciones de la homeostasis entre el sistema nervioso autónomo simpático y parasimpático, y cambios en el metabolismo 29-30.

Una forma de evaluar la funcionalidad del eje $\mathrm{H}-\mathrm{H}-\mathrm{A}$ es a través del test de respuesta del cortisol al despertar (conocido por su sigla en inglés como CAR ${ }^{31}$. La respuesta del cortisol al despertar representa el aumento sustancial del cortisol que ocurre en respuesta a la vigilia, con niveles de cortisol que alcanzan su punto máximo 30-40 min después de la vigilia ${ }^{32}$. Varios autores han propuesto que la RCD bloqueada estaría implicada en la etiología de la depresión ${ }^{33-34}$; sin embargo, otros trabajos contradicen estos hallazgos ${ }^{35-36}$.

Eva Melin et al. hallaron alteraciones del cortisol salival nocturno en pacientes con depresión y $D M 11^{19}$, pero no utilizaron el test de RCD para evaluar la funcionalidad del eje $\mathrm{H}-\mathrm{H}-\mathrm{A}$. Nuestro trabajo es el primero en evaluar el RCD en PD1con y $\sin$ DP.

En el presente estudio evaluamos 79 pacientes con DM1, que tenían en promedio 16 años de evolución, con un bajo porcentaje de complicaciones crónicas (13,7\% en promedio), un alto nivel educativo (59\% educación universitaria) y mayoritariamente con acceso al sistema de salud (58\% obra social o pre-pago). Cabe destacar que a pesar de que los PD1 eran asistidos en centros de atención diabetológica, en promedio presentaron una A1c de $8,3 \%$, muy por encima del objetivo propuesto por la Guía de Recomendaciones de la American Diabetes Association/European Association for the Study of Diabetes (ADA/EASD, 2020) ${ }^{37}$.

De la población estudiada, 60 aportaron muestras aptas para evaluar el test de RCD y hallamos que el $68 \%$ de los PD1 presentó el RCD bloqueado.

El $85 \%$ de los PD1 con depresión presentó el RCD bloqueado vs el $60 \%$ de los no deprimidos, siendo esta diferencia estadísticamente significativa. Esta alteración del eje H-H-A podría ser un biomarcador de depresión en PD1 que explicaría, en parte, el exceso de morbimortalidad cardiovas- 
cular que presentan estos pacientes. Kumari et al. hallaron asociación entre el patrón diurno del cortisol en saliva y la mortalidad de causa cardiovascular y por todas las causas en el estudio Whitehall $\|^{38}$. El $60 \%$ que presentó RDC bloqueado, pero que no DP, podría presentar altos niveles de distrés, relacionado o no a la diabetes, dos situaciones que no han sido analizadas en el presente trabajo y que podrían explicar el bloqueo del RCD.

El 39\% de PD1 evaluados presentó depresión. Esta prevalencia hallada supera datos recientemente publicados en Argentina sobre la prevalencia de depresión en la población general adulta que se halla en el orden del $8,7 \%^{39}$.

Es importante destacar que el $28 \%$ tenía depresión leve y el $11 \%$ presentó depresión moderada-severa, y es este sub-grupo que especialmente requeriría adecuada atención especializada, ya que para mejorar clínicamente necesita recibir tratamiento mixto con psicofármacos y psicoterapia.

Cuando evaluamos el mal control glucémico, basándonos en glucemia mayor a $120 \mathrm{mg} / \mathrm{dl}$ y fructosamina mayor a 285 umol/l, encontramos que los pacientes con DPM/S tenían significativamente un peor control metabólico en relación a los PD1 sin depresión y sin embargo no fue significativa dicha relación con la HbA1c. Como la fructosamina refleja el control glucémico de los últimos 15 días, quizás sea un mejor marcador para evaluar la relación entre el control glucémico y los síntomas depresivos, ya que el PHQ-9 indaga sobre la presencia de los mismos en dicho período. Probablemente por ello Stranberg et al., en un estudio realizado con 319 PD1 adultos, al utilizar HbA1c no hallaron relación entre depresión y peor control glucémico ${ }^{40}$.

Las fortalezas del presente estudio son: 1) muestra multicéntrica nacional; 2) diseño prospectivo; 3) la determinación del cortisol se centralizó en el laboratorio de Endocrinología del Hospital Italiano de Buenos Aires; 4) búsqueda de biomarcadores de depresión en PD1. Como limitaciones mencionamos: 1) no haber evaluado la presencia de distrés relacionado o no a la diabetes, ni haber utilizado el MINIV para descartar enfermedad mental; 2) las muestras de cortisol salival se tomaron en un solo día y cada paciente aportó sólo dos muestras (basal y $30 \mathrm{~min}$ ) cuando hubiera sido ideal muestras de saliva en más de un día por la variabilidad del test dada por las demandas del día; 3) escasa representatividad de pacientes atendidos en el ámbito público y de menores recursos económicos; 4) tamaño de la muestra.

\section{CONCLUSIONES}

En pacientes con DM1 y DP se halló el test de RCD bloqueado en un alto porcentaje. La DP moderada/severa se relacionó con peor control glucémico.

Dado que la depresión comórbida con diabetes se asocia a mayor morbimortalidad cardiovascular, podría utilizarse el test RCD como biomarcador de DP y podría servir para estratificar esta subpoblación de alto riesgo. El uso del cuestionario PHQ-9 en la práctica clínica ayudaría a disminuir el sub-diagnóstico de depresión en el consultorio del especialista en diabetes.

Futuros estudios prospectivos y longitudinales deberán testear qué valor predictivo tienen los biomarcadores de depresión en el mal control glucémico y en la enfermedad cardiovascular en pacientes con DM1 y depresión, y evaluar el costoefectividad de dichos procedimientos.

\section{Agradecimientos}

Al Licenciado en Letras Ignacio Arreses por su contribución en la edición del manuscrito.

\section{BIBLIOGRAFÍA}

1. International Diabetes Federation (2019) IDF Diabetes Atlas, 9th edn. International Diabetes Federation, Brussels, Belgium.

2. Classification and Diagnosis of Diabetes: Standards of Medical Care in Diabetes-2018. Diabetes Care jan 2018; 41 (Suplement 1): S13-S27. doi.org/10.2337/dc18-S002.

3. Rotella F, Mannucci E. Depression as a risk factor for diabetes: meta-analysis of longitudinal studies. J Clin Psychiatry 2013: 74:31-37.

4. Marcus M, Yasamy TM, van Ommeren M. Chisholm D, Saxena S. World Health Organization WHO Department of Mental Health and Substance Abuse. Disponible en: http://www.who. int/mental/ HYPERLINK "http://www.who.int/mental/health/ management/depression/who_paper_depression_wfmh_2012. pdf?ua=1"health/management/depression/who_paper_depression_wfmh_2012.pdf?ua=1.

5. International Diabetes Federation. IDF Diabetes Atlas, 6th edn. Brussels, Belgium: International Diabetes Federation, 2013. Disponible en: http://www.idf.org/diabetesatlas.

6. Anderson RJ, Freedland KE, Clouse RE, Lutsman PJ.The prevalence of comorbid depression in adults with diabetes. Diabetes Care 2001; 24:1069-78.

7. Lloyd CE, RoyT, Nouwen A, Chauhan AM. Epidemiology of depression in diabetes: international and cross-cultural issues. J Affect Disord 2012; 142 Suppl: S22-29.

8. Lloyd CE, Nouwen A, Sartorius N, Ahmed HU, Álvarez A, et al. Prevalence and correlates of depressive disorders in people with type 2 diabetes: results from International Prevalence and Treatment and Diabetes and Depression (INTERPRET-DD) study, a collaborative study carried out in 14 countries. Diabetic Med 2018. DOI: 10.1111/dme.13611.

9. Grey M, Whittemore R, Tamborlane W. Depression in type 1 diabetes in children: natural history and correlates. J Psychosom Res 2002; 53:907-11.

10. Korczak DJ, Pereira S, Koulajian K, Matejcek A, Giacca A.Type 1 diabetes mellitus and major depressive disorder: evidence for biologycal link. Diabetologia 2011; 54:2483-93. 
11. Barnard KD, Skinner TC, Peveler R. The prevalence of co-morbid depression in adults with type 1 diabetes: systematic literature review. Diabet Med 2006; 23:445-448.

12. Simon G, Katon W, Lin E, et al. Diabetes complications and depression as predictors of health services costs. Gen Hosp Psychiatry 2005; 27:344-51.

13. González JS, Esbitt SA. Depression and treatment nonadherence in type 2 diabetes: assesments issues and an integrative treatment approach. Epidemiol Psichiatr Soc 2010 Apr-Jun; 19(2):110-5. Review.

14. Lutsman PJ, Andreson RJ, Freedland KE, de Groot M, Carney RM, Clouse RE. Depression and poor glycemic control: a meta-analytic review of the literature. Diabetes Care 2000 Jul; 23(7):934-42.

15. Badescu SV, Tataru C, Kobylinska L, Georgescu EL, Zahiu DM, Zagrean AM, Zagrean L. The association between diabetes meIlitus and depression. J Med Life 2016 Apr-Jun; 9(2): 120-125.

16. Herder C, Schmitt A, Budden F, Reimer A, Kulzer B, Roden M, HaakT, Hermanns N. Association between pro- and anti-inflammatory cytokines and depressive symptoms in patients with diabetes-potential differences by diabetes type and depression scores. Translational Psychiatry 2017; 7:1. DOI 10.1038/s41398017-0009-2.

17. Chiodini I, Adda G, Scillitani A, et al. Cortisol secretion in patients with type 2 diabetes: relationship with chronic complications. Diabetes Care 2007; 30:83-8.

18. Carvalho LA, Urbanova L, Hamer M, Hackett RA, Lazzarino Al, Steptoe A. Blunted glucocorticoid andmineralocorticoid sensitivity to stress in people with diabetes. Psychoneuroendocrinology 2015; 51:209-18. DOI: 10.1016/j.psyneuen. 2014 09.023. Epub 2014 Sep 30.

19. Melin EO, Thunander M, Landin-Olsson M, Hillman M, Thulesius HO. Depression, smoking, physical inactivity and season independently associated with midnight salivary cortisol in type 1 diabetes. BMC Endocrine Disorders 2014; 14:75. Disponible en: http//www.biomedcentral.com/1472-6823/14/75.

20. Wilhelm I, Born J, Kudielka BM, Schlotz W, Wust S. Is the cortisol awakening rise a response to awakening? Pshychoneuroendocrinology 2007; 32:358.366.

21. Lederbogen F, Hummel J, Fademrecht C, et al. Flattened circadian cortisol rhythm in type 2 diabetes. Exp Clin Endocrinol Amp Diabetes 2011; 119(9):573-575. DOI:0.055/s-0031-1275288.

22. Hackett RA, Steptoe A, Kumari M. Association of diurnal patterns in salivary cortisol with type 2 diabetes in the Whitehall II study. J Clin Endocrinol Metab 2014 Dec; 99(12): 4625-4631.Doi: 10.1210/jc.2014-2459.

23. Kroenke K, Spitzer RL, Williams JB. The PHQ-9: validity of a brief depression severity measure. J Gen Med 2001 Sep; 16(9):606-13.

24. Janssen EP, Kohler S, Stethouwer CD, Schaper NC, et al. The patient health Questionnaire- 9 as a screening tool for depression in individuals with type 2 diabetes mellitus: The Maastricht Study. J Am Geriatr Soc 2016 Nov; 64(11):e201-e6. PubMed PMID: 27783384

25. Lloyd CE, Nouwen A, Sartorius N, Ahmed HU, Álvarez A, et al. Research: Educational and psychological aspects. Prevalence and correlates of depressive disorders in people with type 2 diabetes: results from the International Prevalence and Treatment of Diabetes and depression (INTERPRET-DD) study, a collaborative study carried out in 14 countries. Diabetic $\mathrm{Me}-$ dicine 2018 Jun; 35(6):760-769 DOI: 10.1111/dme 13611.
26. Petrak F, Baumeister HM, Skinner TC, Brown A, Holt RIG. Depression and diabetes: treatment and health-care delivery. Lancet Diabetes Endocrinol 2015; 3:472-485.

27. Diez-Quevedo C, Rangil T, Sánchez-Planell L, Kroenke K, et al. Validation and utility of the patient helath questionnaire in diagnosing mental disorders in 1003 General Hospital Spanish Inpatients. Psychosom Med 2001; 63: 679-86.

28. Van Dooren FEP, Nefs G, Schram MT, Verhey FRJ, Denollet J, Pouwer F. Depression and risk of mortality in people with diabetes mellitus: a systematic review and meta-analysis. PloS ONE 2013; 8(3): e57058. DOI:10.1371/journal.pone.0057058.

29. Roy T, Lloyd CE. Epidemiology of depression and diabetes: a systematic review. J Affect Disord 2012; 142(S1):S8-S21.

30. Ismail K, Sartorius N: Unraveling the pathogenesis of depression diabetes link. In: Depression and diabetes. Edited by Katon W, Maj M. Oxford,UK: Wiley-Blackwell; 2010; 29-62.

31. Clow A, Hucklebridge F, Stalder T, Evans P, Thorn P. The cortisol awakening response: more than a measure of HPA axis function. Neuroscience \& Biobehavioral Reviews 2010; 35 (1):97-103.

32. Clow A, Hucklebridge F, Thorn L. The cortisol awakening response in context. Int Rev Neurobiol 2010; 93:153-75.

33. Vrshek-Schallhorn S, Doane LD, Mineka S, Zinbarg RE, Craske MG, Adam EK. The cortisol awakening response predicts major depression: predictive stability over a 4-year follow-up and effect of depression history. Psychol Med 2013; 4434(3):483-93.

34. Stroud CB, Vrshek-Shallhorn S, Norkett EM, Doane LD. The cortisol awakening response (CAR) interacts with acute interpersonal stress to prospectively predict depressive symptons among early adolescent girls. Psychoneuroendocrinology 2019; 9-18.

35. Adam EK, Doane LD, Zinbarg RE, Mineka S, et al. Prospective prediction of major depressive disorder from cortisol awakening responses in adolescence. Psychoneuroendocrinology 2010 Jul; 35(6):921-31.

36. Vreeburg SA, Hoogendijk WJ, van Pelt J, et al. Major depressive disorder and hypothalamic-pituitary-adrenal axis activity: results from a large cohort study. Arch Gen Psychiatry 2009; 66(6):617-626.

37. American Diabetes Association Glycemic Targets: Standars of Medical Care in Diabetes-2020. Diabetes Care 2020 Jan; 43 (Supplement 1): S66-S76.

38. Kumari M, Shipley M, Stafford M, Kivimaki M. Association of diurnal patterns in salivary cortisol with all-cause and cardiovascular mortality: findings from Whitehall II study. J Clin Endocrinol Metab 2011;96:1478-1485.

39. Stagnaro JC, Cía A, Vázquez N, Vommaro H, et al. Estudio epidemiológico de salud mental en población general de la República Argentina. VERTEX Revista Arg De Psiquiat 2018; Vol. XXIX:275-299.

40. Strandberg RB, Graue M, Wentzel-Larsen T, Peyrot M, Rokne B. Relationships of diabetes-specific emotional distress, depression, anxiety, and overall well-being with $\mathrm{HbA1c}$ in adults persons with type 1 diabetes. J Psychosom Res 2014 Sep;77(3):174-9. 